\title{
Conductance of molecular wires and transport calculations based on density-functional theory
}

\author{
F. Evers, F. Weigend, and M. Koentopp \\ Institut für Nanotechnologie, Forschungszentrum Karlsruhe, 76021 Karlsruhe, Germany
}

(Received 5 December 2003; published 25 June 2004)

\begin{abstract}
The experimental value for the zero bias conductance of organic molecules coupled by thiol-groups to gold electrodes tends to be much smaller than the theoretical result based on density functional theory (DFT) calculations, often by orders of magnitude. To address this puzzle we have analyzed the regime within which the approximations made in these calculations are valid. Our results suggest that a standard step in DFT based transport calculations, namely approximating the exchange-correlation potential in quasistatic nonequilibrium by its standard equilibrium expression, is not justified at weak coupling. We propose, that the breakdown of this approximation is the most important source for overestimating the width of the experimentally observed conductance peak and therefore also of the zero bias conductance. We present a numerical study on the conductance of an organic molecule that has recently been studied in experiments that fully agrees with this conclusion.
\end{abstract}

DOI: 10.1103/PhysRevB.69.235411

PACS number(s): 73.23.-b, 73.40.Cg, 73.63.Rt, 71.15.Mb

\section{INTRODUCTION}

Recently, several conductance measurements of single organic molecules have been reported. ${ }^{1-5}$ Figure 1 illustrates a typical setup with one of the molecules used in experiments. ${ }^{1}$

In part the tremendous attention that this field, "molecular electronics," has received in recent years is due to its possible technological impact. Indeed, a molecular storage device has already been built ${ }^{6}$ and also molecular switches have been realized. ${ }^{7}$ It is clear that for technological applications being able to systematically model and understand the charge transport properties of the combined system of molecule and contacts is of crucial importance.

Since the conductance is very sensitive to details of spectral and orbital properties of molecules and their wave functions, ab initio methods like density functional theory (DFT) are an indispensable tool not only for structure calculations but also for transport theory. Nowadays, DFT calculations including several thousand electrons are possible, which allows the treatment of large molecules and also to include parts of the contacts for more realistic calculations. Despite these enormous capacities, a quantitative description of transport for weakly coupled molecules with a conductance well below the conductance quantum, $g \ll 1$, has not been achieved. In fact, experimental and theoretical values for the zero bias conductance of organic molecules, e.g., benzene, often differ by $1-3$ orders of magnitude. ${ }^{8,9}$

In the present work we consider the organic molecule depicted in Fig. 1, which has been subject to experimental ${ }^{1}$ and DFT based theoretical investigations, ${ }^{10}$ before. In this case the experimental value for the zero bias conductance is smaller than the theoretical one by a factor $\sim 20$. Three different reasons that possibly could lead to this discrepancy should be mentioned and discussed. ${ }^{11}$

First, Fig. 1 shows an idealized situation and actual microscopic conditions realized in the experiment are not known. However, we have chosen the particular case, Fig. 1, because the experimental results are well reproducible. ${ }^{22}$ If important variations in the atomic structure of the contact would exist, one should expect strong fluctuations in the con- ductivity from sample to sample. Indeed fluctuations are present, see Fig. 4 below, but their magnitude is relatively small so that strong structural variations are unlikely to occur. Small variations in structure, on the other hand, tend to have little impact on the theoretical conductance, only. Our calculations show that the transmission is not very sensitive to changes in the bond angles or bond lengths as long as the change is within reasonable limits. Therefore, deviations from the assumed atomic arrangement depicted in Fig. 1 from its experimental realization do not seem to offer a plausible explanation of the large discrepancies observed.

Second, in theoretical calculations approximations have to be done when the molecule is coupled to the leads. Artefacts can be excluded only, when parts of the leads are included into the calculation ("extended molecule"), so that the following hierarchy of inequalities is met:

$$
\delta_{\mathrm{eM}}<\gamma_{\mathrm{eM}} \ll \gamma_{\mathrm{M}}
$$

( $\delta_{\mathrm{eM}}$, level spacing of extended molecule; $\gamma_{\mathrm{M}}\left(\gamma_{\mathrm{eM}}\right)$ level broadening of bare (extended) molecule when coupled to the leads). Indeed, these conditions have not been met in previous calculations, ${ }^{10}$ where $\delta_{\mathrm{eM}} \approx \gamma_{\mathrm{M}}$. In order to improve upon this result, we have performed conductance calculations for the extended molecule, Fig. 1, where $\delta_{\mathrm{eM}} / \gamma_{\mathrm{M}} \approx 0.1$ and $\delta_{\mathrm{eM}} / \delta_{\mathrm{M}} \approx 0.01$ so that the hierarchy of inequalities is satis-

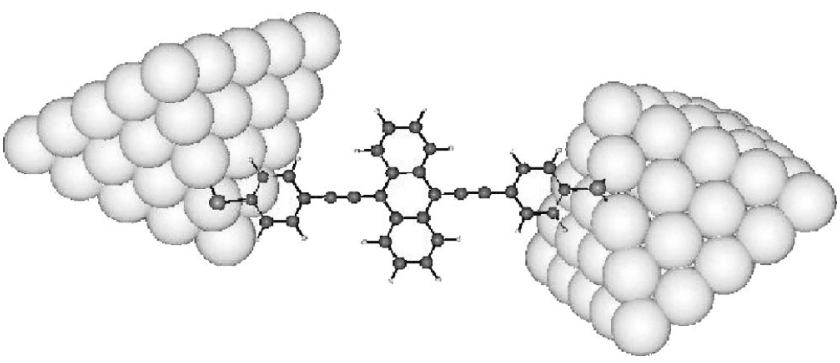

FIG. 1. Schematic representation for a conductance measurement of the molecule $\left(9,10-\operatorname{Bis}\left(\left(2^{\prime}\right.\right.\right.$-para-mercaptophenyl)ethinyl)-anthracene): between gold contacts (Ref. 1). 
fied and artefacts from modeling of the leads can be excluded. We find that qualitative agreement of our data with the experiment is improved significantly as compared to the earlier calculation. ${ }^{10}$ However, the quantitative disagreement is not decreased but rather increased by another factor of 10 ! This shows that insufficient modeling can be ruled out as a source for the discrepancy.

After we have discarded two obvious possibilities for explaining the observed discrepancy, we have to resort to more fundamental considerations. In this paper, we advocate a third possibility, namely that the standard implementation of DFT based transport calculations relies crucially on assumptions that are not justified in the limit of "weak coupling" where the spatial structure of molecular wavefunctions is strongly inhomogenous and transport is dominated by single resonances. ${ }^{12}$

Let us give an outline of our paper and a brief account of key results. In Sec. II we discuss the derivation of the basic equation of DFT transport, Eq. , and show that it has a wide range of applicability. This is true provided that the DFT calculations are performed with an exchange-correlation potential appropriate for the nonequilibrium situation. However, since this potential is not known in general, in practice an "equilibrium exchange correlation approximation" (EXCA) is made in which the standard equilibrium potential is used. A priori, this approximation is uncontrolled. In Sec. II B we introduce an alternative-but equivalentformulation of transport, the Kubo formalism. It enables us to give analytic arguments for the applicability of the EXCA. Our results suggest that the EXCA can be trusted only in the case of nonresonant transport, when the molecular level broadening is strong and individual molecular levels strongly overlap. A typical example for this case is a chain of gold atoms. By contrast, if the transmission exhibits resonances, transport is determined by individual molecular orbitals and their broadening. In this case, the corrections to the EXCA become significant and in particular the level broadening and therefore the zero bias conductance may be severely overestimated. The molecule in Fig. 1 is a representative for these systems.

In Sec. III, we present numerical transport calculations for a gold chain and the molecule, depicted in Fig. 1, that we have already alluded to above. In view of our theoretical analysis we propose that it is the breakdown of the EXCA in the weakly coupled limit that is the cause of the large discrepancy between theoretical and experimental molecular conductance. A discussion of our findings will be given in Sec. IV.

\section{TRANSPORT FORMALISMS}

\section{A. Landauer-Büttiker formalism for interacting electrons}

Meir and Wingreen have derived a general expression for the current flowing through a region of space, where the charge carriers can interact-like a quantum dot or a molecule. ${ }^{13}$ Specifically, the Hamiltonian has the structure

$$
H=H_{0}\left(\left\{\mathbf{d}_{m}^{\dagger}\right\} ;\left\{\mathbf{d}_{m}\right\}\right)+\sum_{\alpha=l, r} \epsilon_{\alpha} \mathbf{c}_{\alpha}^{\dagger} \mathbf{c}_{\alpha}+\sum_{m \alpha=l r}\left(t_{\alpha m} \mathbf{c}_{\alpha}^{\dagger} \mathbf{d}_{m}+\text { H. c. }\right) .
$$

The first term describes the bare molecule which in general may include the electron-electron interaction. Its detailed structure will be of no importance in what follows. The molecule makes contact to two leads denoted left and right. The bare leads are assumed to be noninteracting and described by the second term in Eq. (2). The third term represents the contact.

The Meir-Wingreen formula connects the retarded (advanced) Green's functions $\mathbf{G}\left(\mathbf{G}^{\dagger}\right)$ and the lesser function $\mathbf{G}^{<}$ of the full many-body problem (including the leads) with the dc current,

$$
I=\int d E \operatorname{tr}\left(f_{\mathrm{L}} \boldsymbol{\Gamma}_{\mathbf{L}}-f_{\mathrm{R}} \boldsymbol{\Gamma}_{\mathbf{R}}\right)\left(\mathbf{G}-\mathbf{G}^{\dagger}\right)+\operatorname{tr}\left(\boldsymbol{\Gamma}_{\mathbf{L}}-\boldsymbol{\Gamma}_{\mathbf{R}}\right) \mathbf{G}^{<},
$$

where $f_{\mathrm{L}, \mathrm{R}}=f\left(E-\mu_{\mathrm{R}, \mathrm{L}}\right)$ denotes the Fermi distribution functions for the leads at chemical potentials $\mu_{R, L}$ and $\boldsymbol{\Gamma}_{\mathbf{L}, \mathbf{R}}$ is the imaginary part of the self-energy that describes the coupling of the molecule to the external leads. (For the most part the dependence on energy $E$ will be suppressed in our notation.) For noninteracting particles, in terms of these self-energies we have $\mathbf{G}^{-1}=E \mathbf{1}-\mathbf{H}_{\mathbf{0}}-\Sigma_{\mathbf{L}}-\Sigma_{\mathbf{R}}$, so that $\boldsymbol{\Gamma}_{\mathbf{L}, \mathbf{R}}=i\left(\Sigma_{\mathbf{L}, \mathbf{R}}\right.$ $\left.-\Sigma_{\mathbf{L}, \mathbf{R}}^{\dagger}\right)$ and

$$
\mathbf{G}^{<}=\mathbf{i} \mathbf{G}\left(f_{\mathrm{L}} \boldsymbol{\Gamma}_{\mathbf{L}}+f_{\mathrm{R}} \boldsymbol{\Gamma}_{\mathbf{R}}\right) \mathbf{G}^{\dagger} .
$$

In the appendix we demonstrate that Eq. (4) is just the statement, that the density matrix can be constructed from left and right going scattering states $\psi_{l, r}[x=(\mathbf{x}, t)]$,

$$
G^{<}\left(x, x^{\prime}\right)=\sum_{l, r} f_{l} \psi_{l}(x) \psi_{l}^{*}\left(x^{\prime}\right)+f_{r} \psi_{r}(x) \psi_{r}^{*}\left(x^{\prime}\right) .
$$

From (3) together with (4) we find for the transmission in the limit of linear response and zero temperature

$$
T(E)=\operatorname{tr} \boldsymbol{\Gamma}_{\mathbf{L}} \mathbf{G} \boldsymbol{\Gamma}_{\mathbf{R}} \mathbf{G}^{\dagger} .
$$

Equation (6) is an incarnation of the familiar LandauerBüttiker formula ${ }^{14}$ and valid for noninteracting electrons.

In this section, we argue that expression (6) continues to hold also for a much larger class of interacting problems. This is because according to the Runge-Gross theorem of time dependent DFT (TDDFT) the time evolution of any many-particle Hamiltonian can be calculated by solving a single particle problem in an appropriate effective potential. ${ }^{15}$ Since this point is of importance to us we elaborate on it in the following.

The effective time-dependent single particle problem we should solve is of the form

$$
i \frac{d}{d t} \theta_{n}(x)=\left(-\frac{1}{2 m} \nabla^{2}+V_{s}(x)\right) \theta_{n}(x),
$$

where 


$$
V_{s}(t)=V_{\mathrm{i}}+V_{\mathrm{H}}[n]+V_{\mathrm{XC}}[n](t)+V_{\mathrm{ex}}(t)
$$

with $V_{\mathrm{i}}$ denoting the ion-core potential, $V_{\mathrm{H}}$ the Hartree interaction, $V_{\mathrm{XC}}$ the exchange-correlation potential and $V_{\mathrm{ex}}$ the external probing field. (Notation suppresses the spatial index $\mathbf{x}$.) In addition to the explicit time dependence of the potential $V_{s}$ imposed by $V_{\text {ex }}$ an implicit dependence exists because $V_{s}$ is a functional (in general nonlinear and nonlocal in time and space) of the density $n(\mathbf{x}, t)$. Let us assume that Eq. (7) describes the molecule together with the leads and an external perturbation $V_{\mathrm{ex}}$ that is switched on at $t=t_{0}$ and time independent thereafter,

$$
V_{\mathrm{ex}}(x)=V_{\mathrm{ex}}(\mathbf{x}) \Theta\left(t-t_{0}\right) .
$$

At times prior to $t_{0}$ the system is assumed to be in thermal equilibrium $(T=0)$, so that the reservoirs are characterized by occupation numbers $f_{n}$. Then we have $n(x)=\mathcal{G}_{V}^{<}(x, x)$, with

$$
\mathcal{G}_{V}^{<}\left(x, x^{\prime}\right)=\sum_{n} f_{n} \theta_{n}(x) \theta_{n}^{*}\left(x^{\prime}\right),
$$

where $\theta_{n}(\mathbf{x}, t)$ evolves according to Eq. (7). Moreover, we specialize to the case where $V_{\mathrm{ex}}(\mathbf{x})$ generates a monotonous electrical potential drop from $V_{\text {ex }}=V$ in the asymptotic region of the left lead to $V_{\mathrm{ex}}=0$ in the right lead. In response to the potential drop a current is being generated. After an initial period exhibiting transient behavior, there will be a parametrically wide time interval in which the current and the density are quasistationary. This is precisely the situation for which also Eq. (3) has been derived. ${ }^{13}$ It is only at even much longer times, that the electrochemical potential becomes homogeneous again and the current stops to flow. Formally speaking, we perform the order of limits in which the size of the reservoirs is sent to infinity first and $t_{0} \rightarrow-\infty$, thereafter. We mention that, since we are interested in the long-time behavior only, details of how the external potential is switched on, steplike or adiabatic, are unimportant. The corresponding memory is erased inside the reservoirs. For further discussion see the second section in the Appendix.

Since Eq. (9) describes the exact evolution of the time dependent density we can also calculate the (longitudinal) current density and hence find an exact expression for the conductance. Indeed, the same reasoning that is used for noninteracting electrons to relate transport to a scattering problem can be employed for the present effective single particle problem as well. Consequently, a zero temperature description of the quasistationary region in terms of scattering states should exist with $\mathcal{G}_{V}^{<}$taking a form similar to Eqs. (4) and (5),

$$
\mathcal{G}_{V}^{<}\left(x, x^{\prime}\right)=\sum_{l, r} f_{l} \theta_{l}(x) \theta_{l}^{*}\left(x^{\prime}\right)+f_{r} \theta_{r}(x) \theta_{r}^{*}\left(x^{\prime}\right) .
$$

It involves the Kohn-Sham orbitals $\theta_{r, l}$ representing the scattering states of the quasistationary nonequilibrium situation and their zero temperature occupation numbers $f_{l, r}$ imposed by the left and right reservoirs. Since also the derivation of Eq. (4) can be repeated for an effective single particle problem, a relation analogous to (3) also holds for $\mathcal{G}^{<}$and the corresponding retarded Green's function $\mathcal{G}$. Together with the previous statement (10) this implies that the transmission is given by

$$
T=\operatorname{tr} \boldsymbol{\Gamma}_{\mathbf{L}} \mathcal{G}_{\mathbf{V}} \boldsymbol{\Gamma}_{\mathbf{R}} \mathcal{G}_{\mathbf{V}}^{\dagger}
$$

with $\mathcal{G}_{V}^{-1}(E)=\mathcal{G}_{\mathbf{0 V}}^{-1}-\boldsymbol{\Sigma}_{\mathbf{L}}-\boldsymbol{\Sigma}_{\mathbf{R}}$, and the resolent matrix

$$
\mathcal{G}_{0 V}\left(\mathbf{x}, \mathbf{x}^{\prime}, E\right)=\sum_{n} \frac{\phi_{n}(\mathbf{x}) \phi_{n}^{*}\left(\mathbf{x}^{\prime}\right)}{E-\epsilon_{n}+i \eta / 2}
$$

where the sum is over KS energies $\epsilon_{n}$ and orbitals $\phi_{n}(\mathbf{x})$ calculated for the uncoupled molecule.

Equation (11) constitutes the main result of this section. Similar arguments can also be found in a recent communication by Stefanucci and Almbladh. ${ }^{16}$

Several aspects of our finding should not pass by without further comment.

(a) Equation (11) constitutes the generalization of the Landauer formula to interacting electron systems. In the special case, where $\boldsymbol{\Gamma}_{\mathbf{L}}$ and $\boldsymbol{\Gamma}_{\mathbf{R}}$ differ by a constant factor only, a derivation has already been given by Meir and Wingreen before. ${ }^{13}$ We emphasize, however, that this condition is extremely restrictive. It implies that every atom of the molecule is coupled in precisely the same way to the left lead as it is coupled to the right lead. Given the fact that physical couplings decay with increasing spatial distance, the "condition of proportional couplings" is violated for every realistic system with a finite extent.

(b) As before the self-energies $\boldsymbol{\Sigma}_{\mathbf{L}, \mathbf{R}}$ account for the coupling of the molecule to the leads. It is very well known that they can incorporate sophisticated many body effects, like, i.e., the Abrikosov-Suhl resonance if Kondo-physics is present. ${ }^{17}$ However, one can restructure the problem and define an "extended molecule" that also comprises parts of the contacts, see Fig. 1. The new contact surface $\mathcal{S}_{\mathrm{eM}}$ can be arranged sufficiently far away from the physical contact, so that the new self-energy depends on the type of lead only, but is totally independent of which molecule is used. In fact, if a separation of energy scales exists such that the level spacing of the extended molecule, $\delta_{\mathrm{eM}}$, is much smaller than the broadening, $\gamma_{\mathrm{M}}$, of the bare molecular levels upon coupling to the leads the microscopic information carried by $\boldsymbol{\Sigma}_{\mathbf{L}, \mathbf{R}}$ becomes irrelevant. With any choice of $\gamma_{\mathrm{eM}}$ in accord with Eq. (1) the simple replacement $\Sigma_{\mathbf{L}, \mathbf{R}}\left(\mathbf{x}, \mathbf{x}^{\prime}\right)=i \gamma_{\mathrm{eM}} \delta_{\mathbf{x x}^{\prime}}$ with $\mathbf{x}, \mathbf{x}^{\prime}$ situated on $\mathcal{S}_{\mathrm{eM}}$ is justified. This freedom merely reflects the experimentalists choice to attach leads at convenience from any sort of shape or material as long as the voltage drop is near the real molecule. In this limit, all the detailed information about transport properties of the molecule including correlation effects is carried by the resolvent matrix (11) and inherited from the exchange correlation potential.

(c) Equation (11) has been derived under the condition of vanishing temperature and frequency and in the regime of linear response. Under these restrictions, scattering is energy conserving and therefore an effective single particle scattering formulation of transport can exist. Upon releasing the constraints the incoming particle can exchange energy with the molecule. Qualitatively new phenomena can occur and in general the scattering problem will become much more complicated and may not be understood in terms of a simple 
single particle picture. For example memory effects appear, because here the incoming electron sees the molecule in the state it has been left in after interaction with the previous electrons.

(d) In our arguments we have tacitly assumed that the exchange correlation potential in quasistatic nonequilibrium is independent of time. We give an explicit construction of $V_{\mathrm{XC}}$ valid in the regime of linear response that indeed has this property:

$$
\delta V_{\mathrm{XC}}(\mathbf{r}, t)=\int d \mathbf{r}^{\prime} d t^{\prime} f_{\mathrm{XC}}\left(\mathbf{r}, \mathbf{r}^{\prime}, t-t^{\prime}\right) e^{-\eta\left(t-t^{\prime}\right)} \delta n\left(\mathbf{r}^{\prime}, t^{\prime}\right),
$$

where $\delta$ indicates the deviation from equilibrium and $f_{\mathrm{XC}}\left(x, x^{\prime}\right)=d V_{\mathrm{XC}}(x) / d n\left(x^{\prime}\right) \quad$ denotes the exchangecorrelation kernel that produces a linear density response of the effective KS system which is identical to the true (interacting) density response. The convergence factor $\eta$ has the same meaning as in the usual theories of linear response. It ensures that in the case where the density becomes stationary

$$
\delta V_{\mathrm{XC}}(\mathbf{r})=\int d \mathbf{r}^{\prime} \delta n\left(\mathbf{r}^{\prime}\right) \int d t^{\prime} f_{\mathrm{XC}}\left(\mathbf{r}, \mathbf{r}^{\prime}, t-t^{\prime}\right) e^{-\eta\left(t-t^{\prime}\right)}
$$

the time integral exists so that the asymptotic behavior is well defined.

(e) Equation (12) suggests that near equilibrium (i.e., for the linear response) a variational principle may exist that allows for the calculation of the voltage at a given current or vice versa. The idea is to introduce a density operator that maximizes the entropy under the constraint that the current be finite. Work along this line has been done by $\operatorname{Kosov}^{18}$ and by Delaney and Greer, ${ }^{19}$ recently.

\section{B. Kubo formalism of linear response}

The practical usefulness of Eq. (11) is limited since not much is known about the exchange-correlation potential $V_{\mathrm{XC}}(V)$ that defines the Kohn-Sham problem in quasistatic nonequilibrium. Throughout all previous works using the DFT approach to transport it has been universally assumed that $V_{\mathrm{XC}}(V)$ may be approximated by its equilibrium form $V_{\mathrm{XC}}(0)$ used in standard DFT calculations (EXCA). Let us discuss now, under which conditions this approximation may be expected to hold.

Instead of solving a scattering problem one can also find the conductance from the alternative, but equivalent, Kubo formalism. The advantage of this starting point for our purposes is that analytical statements about the excitation frequencies are available which contain information about the corrections to the EXCA.

The current is related to the dynamical polarization

$$
\mathbf{I}=d \mathbf{P} / d t .
$$

As a consequence the linear current response to a homogeneous external electric field is governed by the dynamical polarizability tensor of the entire system including molecule and leads,

$$
\mathbf{I}=-i \omega \boldsymbol{\alpha}(\omega) \mathbf{E}_{\mathrm{ex}},
$$

which is closely related to the full density susceptibility,

$$
\alpha_{i j}(\omega)=\int d \mathbf{x} d \mathbf{x}^{\prime} x_{i} x_{j}^{\prime} \chi\left(\mathbf{x}, \mathbf{x}^{\prime}, \omega\right),
$$

with the corresponding Lehmann representation

$$
\alpha_{i j}(\omega)=\sum_{s=0} \frac{\left\langle 0\left|\hat{x}_{i}\right| s\right\rangle\left\langle s\left|\hat{x}_{j}\right| 0\right\rangle}{\omega-E_{s 0}+i \eta} .
$$

The sum is over all many-body states with energies $E_{s}$ starting from the ground state $s=0$ and the abbreviation $E_{s 0}=E_{s}$ $-E_{0}$ has been used. In the case of strongly resonant transport the sum is dominated by the contribution of a few poles and the off-resonant current results from the finite lifetime of the corresponding excitations which is encoded in the dipole matrix elements. Clearly, this is the regime in which molecules attached with thiol groups find themselves.

In addition to the bare KS response $\chi_{\mathrm{KS}}\left(\mathbf{x}, \mathbf{x}^{\prime}, \omega\right)$ the full density response contains a piece that describes screening $\left(v_{\mathrm{Q}}=\left|\mathbf{x}-\mathbf{x}^{\prime}\right|^{-1}\right)$

$$
\chi^{-1}(\omega)=\chi_{\mathrm{KS}}^{-1}(\omega)-v_{\mathrm{Q}}-f_{\mathrm{XC}}(\omega) .
$$

In fact, Eq. (17) can be thought of as a definition of $f_{\mathrm{XC}}$ (and hence and $V_{\mathrm{XC}}$ ). The crucial point is that the additional terms in (17) shift the excitation energies $\Omega_{\mu}$ away from their bare KS values $\omega_{i j}^{\mathrm{KS}}=\epsilon_{i}-\epsilon_{j}$ [we introduce a multi-index $\mu=(i, j)$ ],

$$
\Omega_{\mu} \approx \omega_{\mu}^{\mathrm{KS}}+\left\langle\mu\left|v_{\mathrm{Q}}\right| \mu\right\rangle+\left\langle\mu\left|f_{\mathrm{XC}}\left(\omega_{\mu}^{\mathrm{KS}}\right)\right| \mu\right\rangle,
$$

where $|\mu\rangle=|i, j\rangle$ denotes a single particle transition from the occupied KS-orbit $j$ to the vacant orbit $i$. This "single pole approximation" is valid as long as the frequency shift is small compared to the transition frequencies. ${ }^{20}$ We expect this to be a good approximation for those weakly coupled resonant molecular levels that determine the transport through the molecule.

When $f_{\mathrm{XC}}$ is decomposed into its exchange contribution and the correlation part $f_{\mathrm{C}}$, further analytical progress is possible $^{21}$ and one finds

$$
\begin{aligned}
\Omega_{\mu} \approx & \omega_{\mu}^{\mathrm{KS}}+\left\langle j\left|\hat{v}_{\mathrm{x}}^{\mathrm{HF}}-v_{\mathrm{x}}^{\mathrm{KS}}\right| j\right\rangle-\left\langle i\left|\hat{v}_{\mathrm{x}}^{\mathrm{HF}}-v_{\mathrm{x}}^{\mathrm{KS}}\right| i\right\rangle \\
& +\left\langle\mu\left|v_{\mathrm{Q}}\right| \mu\right\rangle-\left\langle i i\left|v_{\mathrm{Q}}\right| j j\right\rangle+\left\langle\mu\left|f_{\mathrm{C}}\right| \mu\right\rangle .
\end{aligned}
$$

$\left[\hat{v}_{\mathrm{x}}^{\mathrm{HF}}\right.$, exchange part of the bare Hartree-Fock (HF) operator; $v_{\mathrm{x}}^{\mathrm{KS}}$, exchange contribution to $V_{\mathrm{XC}}$ in the static KS equation]. Equation (19) contains the plausible statement that the actual excitation energies move away from their KS values closer to their Hartree-Fock estimates when screening is taken into account. ${ }^{21}$ Note, that a corresponding change must be expected to happen also in the dipole matrix elements of Eq. (16) which can be important since they control the lifetime of the excitations.

Equipped with this information we proceed and discuss the validity of the EXCA underlying previous DFT-transport calculations. We distinguish two limiting cases.

(i) Individual resonances strongly overlap, $\gamma_{\mathrm{M}} \gg \delta_{\mathrm{M}}$. This is the limit of strong coupling, $g \sim 1$, where the wavefunctions $|i\rangle$ are extended and the deviations from KS- and HF- 
excitation energies in Eq. (19) are small. In fact, for structureless, plane-wave-like states they vanish. In this case the standard approximation is justified. A chain of Au-atoms provides an exemplary system.

(ii) Individual resonances are fully developed, $\gamma_{\mathrm{M}} \ll \delta_{\mathrm{M}}$. Here, the wave functions show pronounced localized features and in general the corrections to KS-excitation energies and matrix elements will not be small. While qualitatively correct results may still be found in many cases, a systematic quantitative analysis of the resonant structures in $T(E)$ based on the EXCA is not possible, in general. A typical representative of weakly coupled systems is given in Fig. 1. The contact of the extended $\pi$ system to the leads is interrupted by an $S$ atom and therefore the molecular states are partly localized.

\section{NUMERICAL CALCULATIONS}

The discussion presented in the preceding section suggests that the experimental conductance of Au chains is well described by the standard DFT approach whereas the conductance of organic molecules coupled by thiol groups is only poorly captured. In the following section we present transport calculations making use of Eq. (12) together with the EXCA to corroborate this result.

\section{A. Method}

Our method is similar to approaches described in Refs. 23-25, however it has the advantage that using the program package TURBOMOLE we can include a considerably larger number of contact gold atoms. For the molecules of interest to us, this number (110) is sufficient so that the Fermi energy of the extended molecule is very close to the bulk value $E_{F}$ $\approx-5.1 \mathrm{eV}$, even without attaching additional leads.

Since the jest of the method has been outlined previously ${ }^{23-25}$ we can limit ourselves to a brief description. The transmission is given by Eq. (11) but with a resolvent matrix $\mathcal{G}_{0}$ that has been obtained employing the EXCA: KS orbitals and KS energies are taken from a standard DFT calculation. ${ }^{26,27}$ The self-energies, $\boldsymbol{\Sigma}_{\mathbf{L}, \mathbf{R}}$ can be expressed in terms of the hopping matrix elements, $t_{\mathrm{L}, \mathrm{R}}(\mathbf{X} N, \mathbf{x} n)$ which describe a hopping process of an electron in an orbital state $N$ of an atom at position $\mathbf{X}$ of the extended molecule to a state $n$ of the atom at a location $\mathbf{x}$ in the leads: $\boldsymbol{\Sigma}_{\mathbf{L}}=\mathbf{t}_{\mathbf{L}} \mathbf{g}_{\mathbf{L}} \mathbf{t}_{\mathbf{L}}^{\dagger}$ and similarly for $\boldsymbol{\Sigma}_{\mathbf{R}}$. The hopping matrix elements we approximate by their bulk values that we obtain from an independent DFT calculation for a large gold cluster (146 atoms). Likewise, we replace the surface Green's function $\left\langle\mathbf{x} n\left|\mathbf{g}_{\mathbf{L}, \mathbf{R}}\right| \mathbf{x}^{\prime} n^{\prime}\right\rangle$ of the leads by a bulk one taken from the same calculation. ${ }^{28}$

\section{B. Results}

Figure 2 shows our results for the transmission of a linear chain of four equidistantly placed Au atoms $(d=2.67 \AA)$. As a check we have performed calculations with leads that have been modeled by 54 (see Fig. 1) and 84 Au atoms. Moreover the number of "surface" Au atoms from the extended molecule that have been coupled to external leads has been varied: the self-energy has been calculated with 29 and 41 atoms

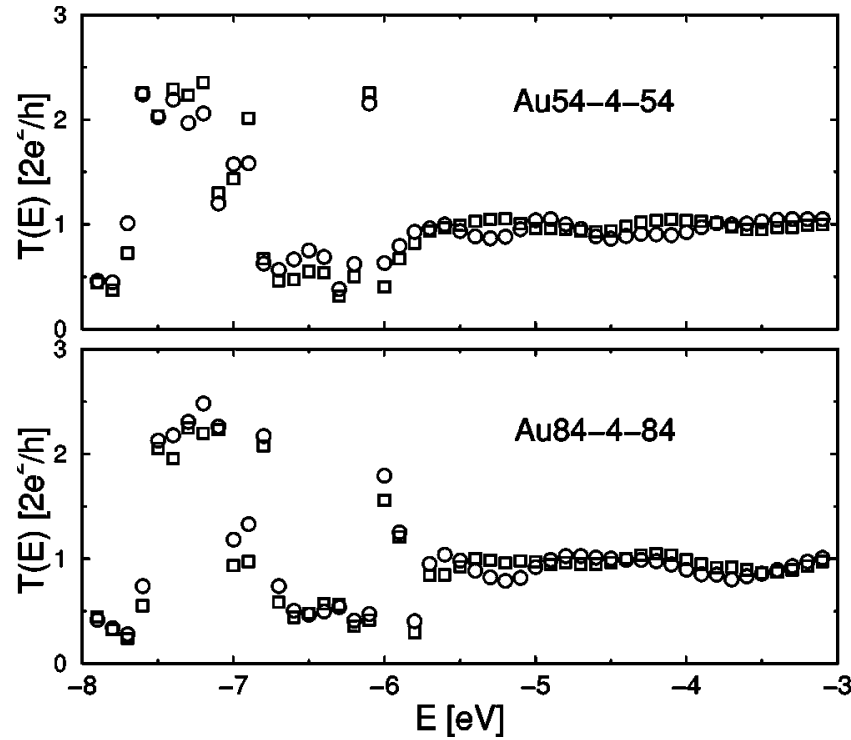

FIG. 2. Transmission of a four atom gold chain. Upper panel, contact was made of 54 gold atoms, see contacts in Fig. 1. Out of these 41 atoms ( $\square, 5 \times 5$ and $4 \times 4$ bottom layers) have been coupled to external leads. For comparison, results with 29 coupling atoms are shown as well $(\circ)$. Lower panel, same as upper panel with 84 gold atoms defining the contacts. $\left(E_{F} \approx-5.1 \mathrm{eV}\right)$ Traces are (nearly) independent of the modeling parameters chosen, and they agree well with experiments and previous calculations (Ref. 29).

taken into account. It can be seen that the transmission is (essentially) independent of these parameters, as it should be. Our results are in very good agreement with experiments and previous calculations. ${ }^{29}$

Before we present our results for the transmission function the coupling of the organic molecule to the contacts should be discussed. In experiments a standard way to facilitate this coupling is to introduce a sulfur atom that forms a very strong bond with gold as well as with carbon atoms. Since the precise microscopic conditions of experiments are not known, several possibilities for the sulfur-gold bonds have to be considered. It is known, that sulfur tends to bind to three gold atoms on a plane $\mathrm{Au}(111)$ surface, i.e., the hollow site is the most stable one. ${ }^{30}$ Binding to just one gold atom-on top position - corresponds to a local minimum of the free energy and binding to two gold atoms is unstable. We find that the situation on rough surfaces exhibiting edges is different. Near an edge, the sulfur finds its most stable position by binding to two gold atoms (Fig. 1) in agreement with an earlier study on much smaller systems. ${ }^{31,32}$ The ontop position remains a minimum albeit at higher energy $(+0.7 \mathrm{eV})$ and the hollow position is unstable. Because in the break junction experiments of interest to us, the sulfur atom is likely to be exposed to an irregular surface, we consider all three cases.

In Figs. 3 and 4 we show the transmission and the $I / V$ characteristics of the molecule of Fig. 1.

The traces correspond to the $\mathrm{S}$ atom binding to one, two or three $\mathrm{Au}$ atoms. These microdeformations induce a slight shift of the transmission peak near $-5.2 \mathrm{eV}$ by about $0.2 \mathrm{eV}$.

Qualitatively, the observed features are in accord with experimental findings of Ref. 1, inset of Fig. 4: we find a con- 


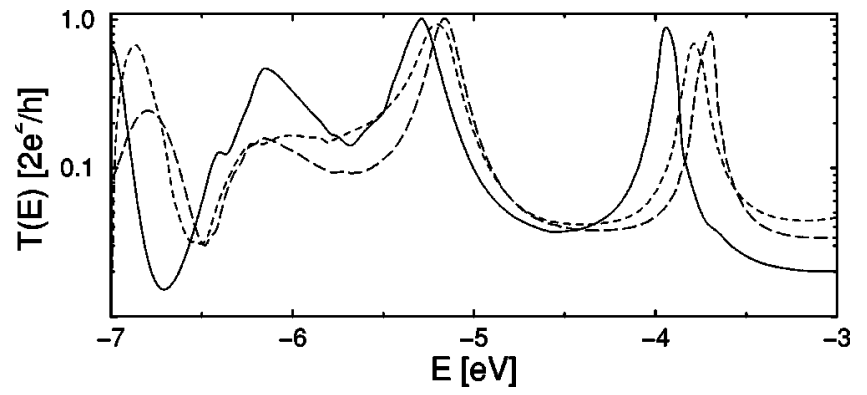

FIG. 3. Transmission of the molecule in Fig. 1 with sulfur atom coupling to one (solid), two (dashed), and three (dotted) gold atoms. $\left(E_{F} \approx-5.1 \mathrm{eV}\right)$ Modifications in the atomic contact structure lead to a slight shift of the conductance peak that offer an explanation for the inhomogeneous peak broadening observed in experiments, see Fig. 4.

ductance gap and the differential conductance exhibits a maximum at about $0.4 \mathrm{~V}$ that stems from a resonant molecular level-the remnant of the HOMO of the bare moleculeabout $0.2 \mathrm{eV}$ below the Fermi energy. The peak conductance and the zero bias conductance can differ by an order of magnitude and at larger voltages the conductance rapidly decreases before there is another increase, again. On the quantitative level serious discrepancies between our calculations and experiments persist: our value for the zero bias conductance $0.2 e^{2} / h$ exceeds the experimental one, $\sim 10^{-3} e^{2} / h$, by more than two orders of magnitude. Moreover, the width of the experimental peak is strongly temperature dependent and can decrease by an order of magnitude with temperature decreasing from $300 \mathrm{~K}$ to $30 \mathrm{~K} .^{22}$ This strongly suggests that the experimental peak width at room temperature is determined by inhomogeneous broadening rather than a strong coupling to the leads.

The inhomogeneous broadening can be understood on the basis of our results in Fig. 3. We have seen above that the position of the HOMO is roughly accounted for by our DFT procedure and it is mainly its damping that is overestimated. Therefore, the shift of the peak position upon changing the coupling of the molecule to the leads is indicative of a mi-

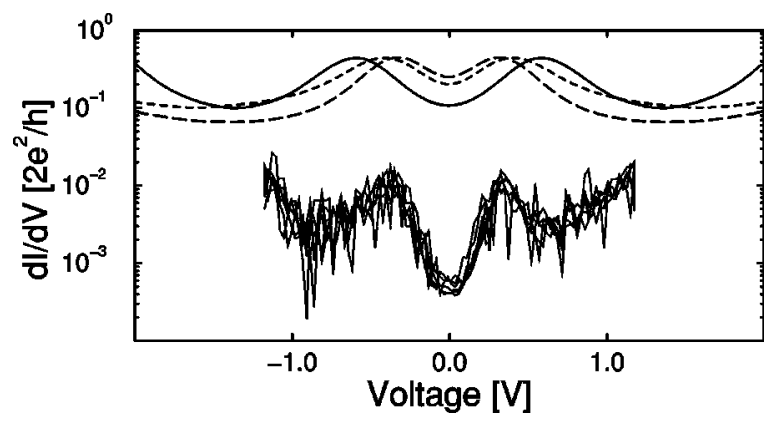

FIG. 4. Upper curves, $d I / d V$ curve from data in Fig. 3 based on $I(V)=\int d E T(E)(f(E+V / 2)-f(E-V / 2))$ with $f(E)$ denoting the Fermi function $(T=300 \mathrm{~K})$. Lower curves, experimental data from Ref. 1. Different traces represent results from consecutive voltage sweeps. The theoretical and experimetal traces exhibit the same qualitative features, but strong quantitative deviations in the conductance magnitude exist.

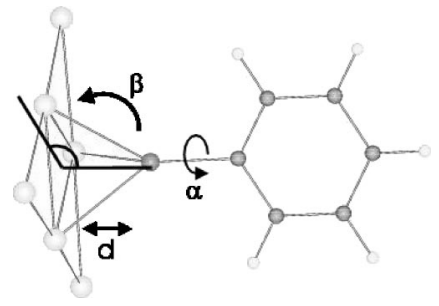

FIG. 5. Schematic representation of geometric degrees of freedom defining the sulfur-gold coupling (center atom, sulfur; left, gold surface; right, benzene molecule).

croscopic smearing mechanism: at sufficiently large temperatures the thermal average over the different types of S-Au bonds leads to an effective broadening on the energy scale $\approx 0.3 \mathrm{eV}$ which indeed is in accord with experimental observations.

One might suspect that at least part of the reason for the large discrepancy between theoretical and experimental findings for the zero bias conductance is that in real experiments the molecule is exposed to various boundary conditions, e.g., stress, that prohibit the contact geometry to relax completely, which is what we have assumed in our calculation. Therefore, an important question is whether the conductance can be strongly affected by a slight change in microscopic degrees of freedom, like the $\mathrm{S}-\mathrm{Au}$ bondlength or the bond angles defined in Fig. 5.

Since our interest is in a qualitative question, we perform our study using the simpler molecule benzene-1,4-dithiol. Our findings for the transmission as a function of energy are in excellent agreement with recent results by Xue and Ratner ${ }^{33}$ and Stokbro et al. ${ }^{34}$ In Fig. 6, upper panel, we display the transmission of this molecule for the geometry op-

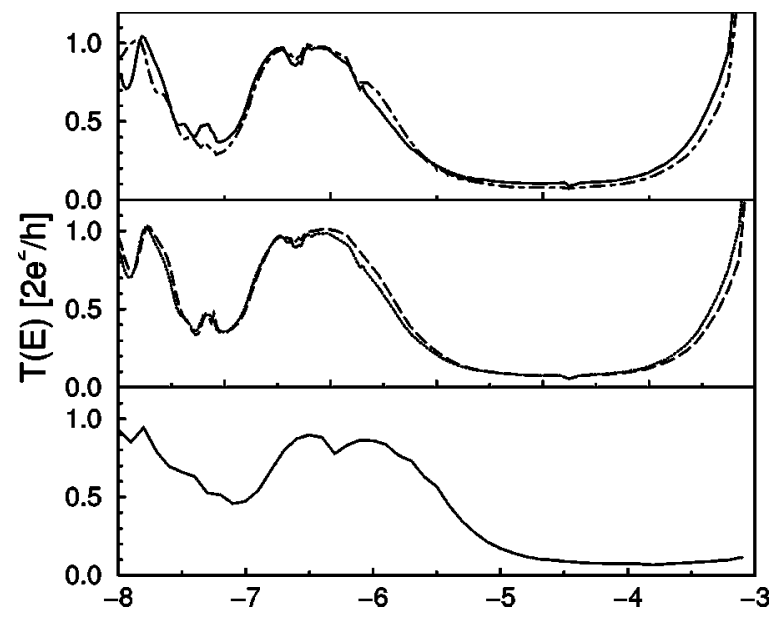

FIG. 6. Transmission of benzene-1,4-dithiol for various parameters $\alpha, d$, and $\beta$ as defined in Fig. 4. Plot shows, that the transmission is robust against small structural modifications induced, e.g., by strain. Upper panel, molecule, coupling to an $\mathrm{Au}(111)$ hollow site, fully geometry optimized (solid) and same molecule after rotation (dotted-dashed, $\alpha=\pi / 24$ ). Middle, change in bondlength (optimal value, $d=2.55 \AA$ ), applied change $0.05 \AA$ (dashed) and $0.1 \AA$ (long dashed). Bottom, change in angle $\beta$ by $\pi / 12\left(E_{F}\right.$ $\approx-5.1 \mathrm{eV})$. 


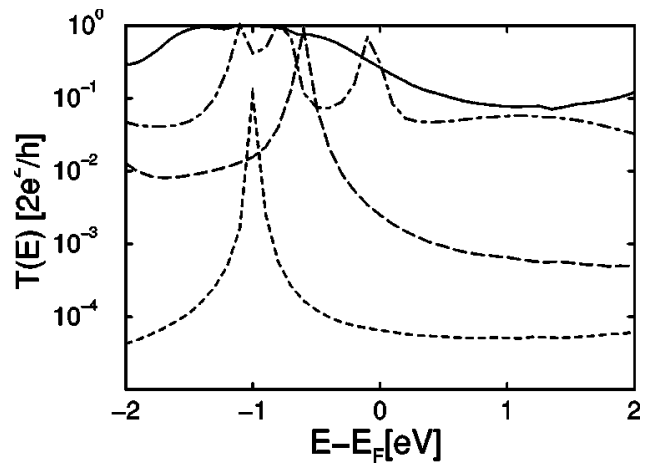

FIG. 7. Transmission of benzene-1,4-dithiol calculated within DFT (solid Au54, as in Fig. 6; dotted-dashed Au14), HF (dashed Au14), and extended Hückel (dotted Au14) with 14 and 54 atom gold clusters simulating the electrodes. DFT and HF calculations show large differences which indicates a breakdown of the EXCA explicated in Sec. II B.

timized case and also after a subsequent rotation of the molecule about angle $\alpha=\pi / 24$ defined in Fig. 4. The middle panel shows the impact of changing the sulfur-gold bondlength $d$ by $0.05 \AA$ and $0.1 \AA$, the lower channel exhibits the change upon changing the bond angle $\beta$ by $\pi / 12$. These manipulations have only a small effect on the transmission confirming earlier findings. ${ }^{33,35}$

The example shows that the DFT conductance does not change by orders of magnitude when changing details of the sulfur-gold bonds within reasonable limits.

Now, we are facing the following situation: On the one hand, the present calculations show that small variations in the nanostructure do not strongly influence the transmission. On the other hand, experiments are reproducible and do not show very strong fluctuations in the zero bias conductance, which implies that large variations in the atomic structure of molecule and contact do not exist. Furthermore, the chemical bond between $\mathrm{S}$ and $\mathrm{Au}$ is known to be very strong and stable-this is why sulfur has been chosen as the coupling element in the first place. Combining these three facts, we conclude that at present there is little evidence that modifications in the atomistic structure of the experimental contacts could exist, which are not properly included in the theoretical modeling, and therefore would hint to an explanation as to why the theoretical conductance exceeds the experimentally measured one so much.

Instead, we advocate another resolution of the puzzle, namely that the mechanism described in Sec. II becomes active because deviations from KS states and HF states are large. To illustrate that the proposed mechanism may indeed induce quantitative changes by orders of magnitude, we compare the transmission calculated within the approximations DFT, HF, and extended Hückel. Figure 7 exhibits the expected behavior, the DFT-transmission peaks are shifted as compared to the Hartree-Fock result and overlap much stronger. In addition, the DFT transmission has a broad background contribution only weakly depending on energy. It stems from electronic states that reside in the leads and "leak" into the molecule. The DFT calculation overestimates tunneling of these states as well. The net result is a difference in the zero bias conductance by two orders of magnitude depending on whether the DFT approximation or the HF approximation is being used. Note, that because of the theoretical argument given in Sec. II B it is not entirely clear, $a$ priori, that it is necessarily DFT that gives the better approximation.

In order to reduce the numerical effort we have restricted ourselves to small electrodes consisting of $14 \mathrm{Au}$ atoms each. By comparing the DFT results for Au14 and Au54 depicted in Fig. 6 one can be convinced, that this simplification leaves unaffected our main conclusion.

\section{CONCLUSIONS}

The numerical results presented in the preceding section are in full agreement with expectations based on the theoretical analysis performed in Sec. II: while the conductance of an Au chain agrees well with experimental results, large quantitative discrepancies for organic molecules attached to gold electrodes via a thiol bond exist. Arguments have been presented to the extent that these discrepancies cannot plausibly be explained by insufficient modeling.

The standard DFT approach can be exact only in the case, where the ground state is represented by a single Slater determinant which in turn implies that $\mathrm{HF}$ is exact. For the molecule this is not the case, however. Since an approximation for the ground state based on the Slater determinant of $\mathrm{KS}$ orbitals is a mixture of the wave function of the exact ground state with its excitations, the character of the approximate state tends to be too delocalized. Consequently, the molecule-lead coupling comes out too strong and the level broadening is overestimated. We have given analytical arguments in favor of this picture.

In order to improve upon the standard DFT approach to transport the exchange correlation potential should be replaced by the appropriate potential for the quasistatic nonequilibrium in order to include the corrections due to dynamical screening that are ignored otherwise. Unfortunately, at present such a potential is not known. However, our work suggests an alternative approach which is based on the Kubo formula. It enables us to to calculate the dynamical polarization of the molecule together with parts of the leads. Thereof we can obtain the dc current in the limit of zero frequency. Work in this direction is under way.

Finally, let us emphasize that our results have implications for the calculation of nonequilibrium effects ${ }^{24}$ like the polarization of those electronic states that do not carry the current. Two effects that work in opposite directions occur. For a given current the voltage drop at the molecule is underestimated by DFT-based calculations (since $g$ is too large) and therefore so is the induced polarizing field. On the other hand, the charge response of localized states to this field is overestimated since they appear too metallic. Consequently, interpreting DFT-based transport calculations for nonequilibrium effects is not straightforward and possible asymmetries in the $I / V$ characteristics could be blurred. ${ }^{36,37}$

\section{ACKNOWLEDGMENTS}

We thank R. Ahlrichs, F. Furche, A. Mildenberger, R. Narayanan, J. Reichert, H. B. Weber, and in particular K. 
Burke and P. Wölfle for useful discussions. Also we thank A. D. Mirlin for a discussion of the "condition of proportional couplings" and M. Wegewijs for providing us with a copy of Ref. 37. Finally, we express our gratitude to J. Reichert and $\mathrm{H}$. Weber for supplying us with the experimental data.

\section{APPENDIX}

\section{Green's functions and scattering states}

We derive Eq. (4) from Eq. (5). The effective single particle Hamiltonian of the scattering problem is of the form $\mathbf{H}=\mathbf{H}_{0}+\mathbf{H}^{\prime}$, where

$$
\mathbf{H}_{0}=\left(\begin{array}{ccc}
h_{L} & 0 & 0 \\
0 & h_{C} & 0 \\
0 & 0 & h_{R}
\end{array}\right), \quad \mathbf{H}^{\prime}=\left(\begin{array}{ccc}
0 & t_{\mathrm{L}}^{\dagger} & 0 \\
t_{\mathrm{L}} & 0 & t_{\mathrm{R}} \\
0 & t_{\mathrm{R}}^{\dagger} & 0
\end{array}\right) .
$$

The Hamiltonian of the uncoupled left (right) lead has been denoted by $h_{L}\left(h_{R}\right)$, the Hamiltonian of the central unit (molecule) is $h_{C}$ and the matrices $t_{\mathrm{L}, \mathrm{R}}$ are the couplings of the left and right leads to the central unit. If the molecule is uncoupled, the scattering states are of the form

$$
\Phi_{l}=\left(\phi_{l}, 0,0\right), \quad \Phi_{r}=\left(0,0, \phi_{r}\right) .
$$

When the coupling has been switched on, the new scattering states $\Psi_{l, r}$ can be obtained from a Lippmann-Schwinger equation,

$$
\Psi_{l, r}=\Phi_{l, r}+\mathcal{G}(E+i 0) \mathbf{H}^{\prime} \Phi_{l, r},
$$

where $E$ labels the energy of the state and the resolvent matrix $\mathcal{G}(z)=[z-\mathbf{H}]^{-1}$ has been introduced. The lesser function is defined by $[x=(\mathbf{x}, t)]$

$$
\mathcal{G}^{<}\left(\mathbf{x}, \mathbf{x}^{\prime}, E\right)=\sum_{\alpha=l, r} \int d\left(t-t^{\prime}\right) \Psi_{\alpha}(x) \Psi_{\alpha}^{*}\left(x^{\prime}\right) f_{\alpha} e^{i E\left(t-t^{\prime}\right)} .
$$

Using Eq. (A3) a matrix representation can be derived

$$
\mathcal{G}^{<}\left(\mathbf{x}, \mathbf{x}^{\prime}, E\right)=\left\langle\mathbf{x}\left|\left[\mathbf{1}+\mathcal{G} \mathbf{H}^{\prime}\right] \mathbf{g}_{\mathbf{0}}^{<}\left[\mathbf{1}+\mathbf{H}^{\prime} \mathcal{G}^{\dagger}\right]\right| \mathbf{x}^{\prime}\right\rangle
$$

with

$$
\mathbf{g}_{\mathbf{0}}^{<}=\left(\begin{array}{ccc}
g_{L}^{<} & 0 & 0 \\
0 & 0 & 0 \\
0 & 0 & g_{R}^{<}
\end{array}\right)
$$

and

$$
g_{L}^{<}\left(\mathbf{x}, \mathbf{x}^{\prime}, E\right)=\sum_{l} \int d\left(t-t^{\prime}\right) \phi_{l}(x) \phi_{l}^{*}\left(x^{\prime}\right) f_{l} e^{i E\left(t-t^{\prime}\right)}
$$

and a similar equation for $g_{R}^{<}$. In Eq. (3) only those matrix elements of $\mathcal{G}^{<}$are needed, that connect the left and right boundaries of the central unit. These elements are given by the term in Eq. (A5) quadratic in the external coupling. Since

$$
\mathbf{H}^{\prime} \mathbf{g}_{\mathbf{0}}^{<} \mathbf{H}^{\prime}=\left(\begin{array}{ccc}
0 & 0 & 0 \\
0 & \Sigma_{C}^{<} & 0 \\
0 & 0 & 0
\end{array}\right)
$$

with $\Sigma_{\mathrm{C}}^{<}=t_{\mathrm{L}} g_{\mathrm{L}}^{<} t_{\mathrm{L}}^{\dagger}+t_{\mathrm{R}} g_{R}^{<} t_{\mathrm{R}}^{\dagger}$ and since $\mathcal{G}_{\mathbf{0}}$ is diagonal one has for the matrix element representing the central unit

$$
\mathcal{G}^{<}=\mathcal{G} \Sigma_{\mathrm{C}}^{<} \mathcal{G}^{\dagger}
$$

and thus recovers Eq. (4). A similar derivation has also been given by Brandbyge et al. ${ }^{24}$

\section{Quasistationary nonequilibrium}

The general conditions under which a nonequilibrium state can be quasistationary are not easily understood. In fact, the density need not become quasistationary if conserved quantities exist that preserve the memory of the switch-on process. To give an example, we consider the situation of quasistatic current flow from a left to a right reservoir that we have introduced in Sec. II A. Immediately after switching on the external voltage the charge flow sets in. It is accompanied by a propagating density mode which is a consequence of momentum conservation and prevents the density to become time independent in the entire system in a strict sense.

However, driving at a formulation of transport in terms of scattering states, what is of primary concern to us is whether the occupation of the incoming waves with wave number $\mathbf{k}$ can be approximated by a Fermi-Dirac distribution $f_{\mathbf{k}}$ characterized by a time-independent temperature and chemical potential $\mu$. This is certainly the case, if the electrons in the leads are noninteracting, since then the incoming waves are decoupled from the outgoing ones.

If the electrons in the leads may not be thought of as noninteracting the situation is more complicated: the outgoing, propagating modes interact with the incoming scattering waves and in fact may change their distribution $f_{\mathbf{k}}$. However, in the presence of dissipation, e.g., electron-phonon interaction, the propagating modes will eventually become diffusive and the system reaches a local thermal equilibrium. Now, the basic assumption underlying our scattering description is that the conductivity of the electrodes is so large that the essential voltage drop occurs only in the vicinity of the molecule with its contacts. This implies then, that the chemical potential $\mu$ is nearly homogeneous within the electrodes.

The crucial point of this scenario is, that it describes a situation in which the occupancy of a scattering state in the leads is given by the equilibrium distribution at a timeindependent chemical potential and temperature. In the absence of dissipation one might suspect, that the interaction between the outgoing and incoming scattering states could be strong enough, in order to drive the occupation of incoming waves away from equilibrium. This would imply an effect on the current to first order in the applied voltage difference and hence modify the conductance. The theoretical analysis of such a mechanism is beyond the scope of the present work. On the other hand, the effect of the current flow on the transmission proper may safely be ignored since it is of higher than linear order in the voltage. 
${ }^{1}$ J. Reichert, R. Ochs, D. Beckmann, H. B. Weber, M. Mayor, and H. v. Löhneysen, Phys. Rev. Lett. 88, 176804 (2002).

2 M. A. Reed, C. Zhou, C. J. Muller, T. P. Burgin, and J. M. Tour, Science 278, 252 (1997); C. Kergueris, J.-P. Bourgoin, S. Palacin, D. Esteve, C. Urbina, M. Magoga, and C. Joachim, Phys. Rev. B 59, 12505 (1999).

${ }^{3}$ X. D. Cui, A. Primak, X. Zarate, J. Tomfohr, O. F. Sankey, A. L. Moore, T. A. Moore, D. Gust, G. Harris, and S. M. Lindsay, Science 294, 871 (2001).

${ }^{4}$ J. Park, A. N. Pasupathy, J. I. Goldsmith, C. Chang, Y. Yaish, J. R. Petta, M. Rinkoski, J. P. Sethna, H. D. Abruna, P. L. McEuen, and D. C. Ralph, Nature (London) 417, 722 (2002).

${ }^{5}$ W. Liang, M. P. Shores, M. Bockrath, J. R. Long, and H. Park, Nature (London) 417, 725 (2002).

${ }^{6}$ K. M. Roth, N. Dontha, R. B. Dabke, D. T. Gryko, C. Clausen, J. S. Lindsey, D. F. Bocian, and W. G. Kuhr, J. Vac. Sci. Technol. B 18, 2359 (2000).

${ }^{7}$ J. Chen and M. A. Reed, Chem. Phys. 281, 127 (2002).

${ }^{8}$ S. T. Pantelides, M. Di Ventra, and N. D. Lang, Ann. N.Y. Acad. Sci. 960, 177 (2002).

${ }^{9}$ E. G. Emberly and G. Kirczenow, Phys. Rev. Lett. 87, 269701 (2001).

${ }^{10}$ J. Heurich, J. C. Cuevas, W. Wenzel, and G. Schön, Phys. Rev. Lett. 88, 256803 (2002).

${ }^{11}$ See also, e.g., A. W. Ghosh and S. Datta, cond-mat/0303630v1.

${ }^{12} \mathrm{Also}$, the KS potential is not known exactly which introduces an additional problem, the "self-interaction problem" [see e.g., R. M. Dreizler and E. K. U. Gross, Density Functional Theory (Springer, Heidelberg, 1990)]. It leads to an estimate for the HOMO-LUMO-gap too small which implies a behavior too metallic. However, the effect depends on the extension of the wave functions and we expect that it is not of primary importance for molecules with extended $\pi$ systems like those in Fig. 1.

${ }^{13}$ Y. Meir and N. S. Wingreen, Phys. Rev. Lett. 68, 2512 (1992).

${ }^{14}$ Y. Imry, Introduction to Mesoscopic Physics (Oxford University Press, Oxford, 2002).

15 E. Runge and E. K. U. Gross, Phys. Rev. Lett. 52, 997 (1984); for a recent review see, e.g., G. Onida, L. Reining, and A. Rubio, Rev. Mod. Phys. 74, 601 (2002).

${ }^{16}$ G. Stefanucci and C.-O. Almbladh, cond-mat/0310084v2.

${ }^{17}$ See, e.g., G. D. Mahan, Many-Particle-Physics, 3rd ed. (Kluwer Academic, New York, 2000).

${ }^{18}$ D. S. Kosov, J. Chem. Phys. 119, 1 (2003).
${ }^{19}$ P. Delaney and J. C. Greer, cond-mat/0312522.

${ }^{20}$ H. Appel, E. K. U. Gross, and K. Burke, Phys. Rev. Lett. 90, 043005 (2003).

${ }^{21}$ X. Gonze and M. Scheffler, Phys. Rev. Lett. 82, 4416 (1999).

${ }^{22}$ J. Reichert, H. B. Weber, M. Mayor, and H. v. Löhneysen, condmat/0212272v1.

${ }^{23}$ P. S. Damle, A. W. Gosh, and S. Datta, Phys. Rev. B 64, 201403 (2001).

${ }^{24}$ M. Brandbyge, J-L. Mozos, P. Ordejon, J. Taylor, and K. Stokbro, Phys. Rev. B 65, 165401 (2002).

${ }^{25}$ Y. Xue, S. Datta, and M. A. Ratner, Chem. Phys. 281, 151 (2002).

${ }^{26}$ O. Treutler and R. Ahlrichs, J. Chem. Phys. 102, 346 (1995); K. Eichkorn, O. Treutler, H. Ohm, M. Haser, and R. Ahlrichs, Chem. Phys. Lett. 240, 283 (1995).

27 We use the BP86-functional;A. D. Becke, Phys. Rev. A 38, 3098 (1988); J. P. Perdew, Phys. Rev. B 33, 8822 (1986).

${ }^{28}$ F. Evers, F. Weigend, and M. Koentopp (unpublished).

29 K. Hansen, S. K. Nielsen, M. Brandbyge, E. Laesgsgaard, I. Stensgaard, and F. Besenbacher, Appl. Phys. Lett. 77, 708 (2000); C. Untiedt, A. I. Yanson, R. Grande, G. Rubio-Bollinger, N. Agrait, S. Vieira, and J. M. van Ruitenbeek, Phys. Rev. B 66, 085418 (2002); J. J. Palacios, A. J. Perez-Jimenez, E. Louis, E. SanFabian, and J. A. Verges, ibid. 66, 035322 (2002); M. Brandbyge, N. Kobayashi, and M. Tsukada, Phys. Rev. B 60, 17064 (1999).

${ }^{30}$ H. Sellers et al., J. Am. Chem. Soc. 115, 9389 (1993).

${ }^{31}$ J. R. Reimers et al., Ann. N.Y. Acad. Sci. 960, 100 (2002).

${ }^{32}$ H. B. Weber, J. Reichert, F. Weigend, R. Ochs, D. Beckmann, M. Mayor, R. Ahlrichs, and H. v. Löhneysen, Chem. Phys. 281, 113 (2002).

${ }^{33}$ Y. Xue and M. A. Ratner, cond-mat/0303179v1.

${ }^{34}$ K. Stokbro, J. Taylor, M. Brandbyge, J. L. Mozos, and P. Ordejon, Comput. Mater. Sci. 27, 151 (2003).

${ }^{35}$ S. N. Yaliraki, M. Kemp, and M. A. Ratner, J. Am. Chem. Soc. 121, 3428 (1999).

${ }^{36}$ K. Stokbro, J. Taylor, and M. Brandbyge, J. Am. Chem. Soc. 125, 3674 (2003).

${ }^{37}$ For a simple model system (quantum dot with on-site interaction plus leads) analytical calculations can be performed and compared with DFT. Consistent with our assertions, large deviations in the strongly nonlinear regime have been found, see $\mathrm{C}$. Romeike, Diploma thesis, RWTH Aachen, 2002. 